\title{
Effect of Validamycin A on the Inositol Content and Branching of Rhizoctonia cerealis and Other Fungi
}

\author{
By GEOFFREY D. ROBSON, ${ }^{1 *}$ PAUL J. KUHN ${ }^{2}$ AND \\ ANTHONY P. J. TRINCI ${ }^{1}$

\begin{abstract}
'Microbiology Group, Department of Cell and Structural Biology, School of Biological Sciences, Stopford Building, University of Manchester, Manchester M13 9PT, UK

${ }^{2}$ Shell Research Limited, Sittingbourne Research Centre, Sittingbourne, Kent ME9 8AG, UK
\end{abstract}

(Received 26 September 1988; accepted 5 December 1988)

\begin{abstract}
The effects of the antibiotic validamycin $\mathrm{A}$ on the inositol content and hyphal branching of Rhizoctonia cerealis were investigated. Inositol was found associated with the cell wall and as a lipid extractable fraction composed of phospholipid, phosphosphingolipid and glycolipid. Validamycin A decreased the inositol content of the phospholipid fraction by $57 \%$ and the phosphosphingolipid fraction by $40 \%$, but had no effect on the glycolipid fraction. The phosphatidylinositol (the only inositol-containing phospholipid detected) content of the biomass was reduced by $53 \%$ in the presence of validamycin $\mathrm{A}$ and this decrease was compensated for by rises in the levels of phosphatidylserine and an unknown, minor phospholipid. Over the concentration range 1 to $5000 \mu \mathrm{M}$, validamycin A caused decreases in the colony radial growth rate, internode length (the distance between adjacent branches on a leading hypha) and inositol content of $R$. cerealis colonies, and a positive correlation was found between inositol content and internode length. Addition of inositol to the medium only slightly antagonized the effect of the antibiotic on mycelial morphology and did not restore phosphatidylinositol levels of the biomass to control values.
\end{abstract}

\section{INTRODUCTION}

Validamycin A is used in Japan and China to control Rhizoctonia solani, the causal agent of rice sheath blight. The antibiotic appears to control the disease due to its paramorphogenic (Tatum et al., 1949) effect on the mould; it decreases the maximum rate of hyphal extension and increases hyphal branching without affecting the organism's specific growth rate (Nioh \& Mizushima, 1974; Trinci, 1984, 1985; Robson et al., 1988). Thus, the spatial distribution of the pathogen is changed by validamycin A (it forms highly colonized mycelia which expand more slowly than control colonies), and this leads to a reduction in the rate of lesion development and restricts the pathogen to the basal parts of infected plants (Wakae \& Matsuura, 1975). Studies on the mode of action of the antibiotic revealed that it did not affect protein or nucleic acid synthesis (Nioh \& Mizushima, 1974). However, validamycin A greatly reduced the amount of inositol in the supernatant of cultures of $R$. solani (Wakae \& Matsuura, 1975). Further studies revealed that the percentage of cucumber seeds which germinated when sown onto the margin of colonies of $R$. solani increased when the medium contained validamycin $\mathrm{A}$, but when the medium also contained inositol there was a decrease in seed germination (Wakae \& Matsuura, 1975). Thus, validamycin A caused a reduction in the inositol content of the supernatant of cultures of $R$. solani and this decrease was correlated with a reduction in the pathogenicity of the fungus. Reversal of the controlling effect of validamycin A in the cucumber seed trials by

Abbreviations: PC, phosphatidylcholine; PI, phosphatidylinositol; PS, phosphatidylserine. 
exogenous inositol was attributed to the structural similarity between inositol and the valienamine and validamine components of the validamycin A molecule (Wakae \& Matsuura, 1975). However, addition of inositol to culture medium failed to reverse the effect of the antibiotic on the morphology of $R$. solani or $R$. cerealis (Shibata et al., 1980, 1981; Trinci, 1984).

When mycelia of inositol auxotrophs of Neurospora crassa are grown on inositol-deficient medium they branch more profusely than parental mycelia and this difference has been correlated with differences in the composition of inositol-containing lipids and cell walls of the mutant and parental mycelia. This observation has led to the hypothesis that inositol plays an important role in regulating hyphal branching (Fuller \& Tatum, 1956; Shatkin \& Tatum, 1961; Hanson \& Brody, 1979). The aim of the present study was to investigate the effect of validamycin A on the inositol metabolism of $R$. cerealis and to try to correlate changes in inositol content of validamycin A treated mycelia with the effect of the antibiotic on hyphal branching.

\section{METHODS}

Organismis and media. Rhizoctonia cerealis (strain R80/101) was obtained from the Plant Breeding Institute, Cambridge, UK. Bjerkandera adusta, Heterobasidion annosum, Phlebia radiata, Phlebia rufa and Pseudotrametes gibbosa were obtained from Dr A. D. N. Rayner (School of Biological Sciences, University of Bath, Bath, UK). Agaricus silvicola, Cystoderma pereniciosa, Daedalia quercina, Flammulina velutipes, Lentinus edodes and Pleurotus ostreatus were obtained from Dr J. N. Hedger (Department of Botany and Microbiology, University College of Wales, Aberystwyth, UK). All other fungi were obtained from the Culture Collection of the Department of Cell and Structural Biology, University of Manchester. All organisms were maintained at $4{ }^{\circ} \mathrm{C}$ on malt extract agar (Lab M) slopes under paraffin oil.

Fungi other than Basidiomycotina were grown on Vogel's (1956) medium containing 5 mM-glucose, $50 \mathrm{~mm}$ glucose or $50 \mathrm{~mm}$-mannitol as the carbon source and the media were prepared as described previously (Robson $e t$ al., 1987). For Basidiomycotina, Vogel's medium was amended with 'growth factors' $(1 \%, \mathrm{v} / \mathrm{v})$ from yeast extract as described by Robson et al. (1988). Medium containing these 'growth factors' will henceforth be referred to as modified Vogel's medium. Inositol ( $1.0 \mathrm{M})$ and validamycin A $(0.1 \mathrm{M})$ were prepared as filter-sterilized (Whatman, $0.22 \mu \mathrm{m})$ aqueous stock solutions, and were added to media to give the final concentrations required. When necessary, media were gelled with $15 \mathrm{~g}$ Taiyo technical grade agar (Davis Gelatine) per litre of medium.

Inoculation and cultural conditions. Fungi were grown on agar in $9 \mathrm{~cm}$ diam. Petri dishes containing $20 \mathrm{ml}$ volumes of media; these were inoculated with $4.5 \mathrm{~mm}$ diam. plugs taken from the margin of fungal colonies in the linear phase of growth. The medium was sometimes overlaid with circles $(8.5 \mathrm{~cm}$ diam.) of Cellophane (Rayophane, British Cellophane); Cellophane was boiled twice in distilled water to remove the plasticizers before being sterilized in water at $121^{\circ} \mathrm{C}$ for $15 \mathrm{~min}$. All cultures were incubated at $25^{\circ} \mathrm{C}$. For inositol determinations, fungi were either grown in stationary thin-layer liquid cultures in Petri dishes containing $10 \mathrm{ml}$ volumes of agar media or in shake flask (200 r.p.m. on a rotary shaker) cultures containing $50 \mathrm{ml}$ volumes of media in $250 \mathrm{ml}$ conical flasks (Robson et al., 1988). Liquid cultures were inoculated with spore suspensions or mycelial blends (Robson et al., 1988).

Growth and morphology. Colony diameters were measured with a Shadowmaster (Baty \& Co., Burgess Hill, Sussex, UK) using a $\times 10$ objective. Dry weights of $R$. cerealis grown on agar were obtained by washing colonies off the Cellophane discs onto pre-dried, weighed filter papers (Whatman no. 1); mycelia grown in liquid culture were also collected onto filter papers. The harvested biomass was washed with $100 \mathrm{ml}$ distilled water and dried at $60^{\circ} \mathrm{C}$ to constant weight. Internode lengths (the distance between adjacent branches on leading hyphae) were measured using a travelling micrometer eyepiece (Olympus) on the first three internodes of leading hyphae at the margin of colonies of $R$. cerealis in the linear phase of growth; internode length provides an indicator of hyphal branching.

Inositol extraction and bioassay. Inositol was liberated from samples by hydrolysis in $4 \mathrm{ml} 6 \mathrm{M}-\mathrm{HCl}$ at $100^{\circ} \mathrm{C}$ in a heating block (Grant BT 5). Biomass and lipid extracts were hydrolysed for 40 to $44 \mathrm{~h}$ and culture supernatants were hydrolysed for 20 to $25 \mathrm{~h}$; these were found to be the optimal times for inositol release. After hydrolysis, samples were dried overnight at $40^{\circ} \mathrm{C}$ on watch glasses, dissolved in water and the process repeated a further two times to remove all traces of acid. The inositol content of samples was determined by bioassay using the method of Paranjapye et al. (1964), except that $4 \mathrm{ml}$ volumes of minimal yeast medium and $1 \mathrm{ml}$ volumes of sterile sample (sterilized at $115^{\circ} \mathrm{C}$ for $10 \mathrm{~min}$ ) in boiling tubes were inoculated with Schizosaccharomyces pombe and incubated on a rotary shaker $\left(250\right.$ r.p.m.) at $30^{\circ} \mathrm{C}$ for 36 h. Three replicate cultures were prepared per sample and optical densities of the cultures were read at $540 \mathrm{~nm}$ against a distilled water blank. Inositol contents of the samples were determined from a standard curve prepared over the concentration range 0 to $2 \mu \mathrm{g}$ inositol $\mathrm{ml}^{-1}$.

Glycerophospholipid extraction and fractionation. Lipids were extracted from biomass using $10 \mathrm{ml}$ ethanol/water/ diethyl ether/pyridine/ammonia (15:15:5:1:0.018, by vol.) at $60^{\circ} \mathrm{C}$ according to Angus \& Lester (1972). The 


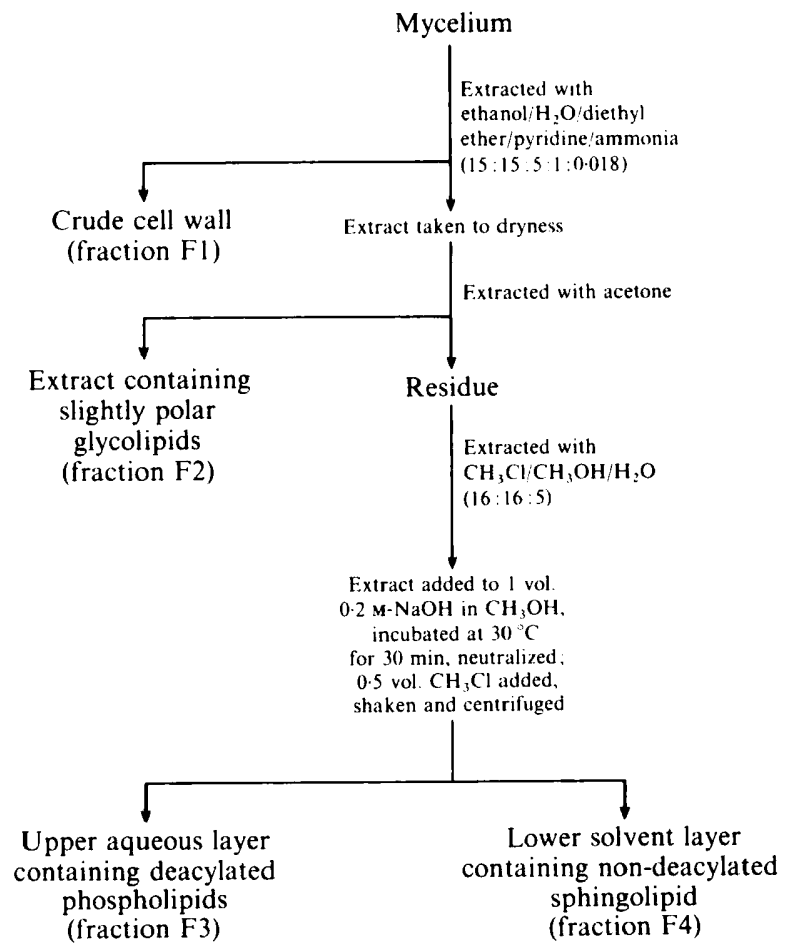

Fig. 1. Procedure for fractionation of inositol from the mycelium of $R$. cerealis.

extract was taken to dryness in vacuo at $45^{\circ} \mathrm{C}$ and the glycerophospholipids were extracted from the residue in $2 \mathrm{ml}$ chloroform/methanol $(2: 1, \mathrm{v} / \mathrm{v})$; the extraction procedure was repeated three times for each residue. The chloroform/methanol extracts from each sample were combined, transferred to glass vials, taken to dryness under a stream of nitrogen and stored at $-20^{\circ} \mathrm{C}$.

Glycerophospholipid separation, identification and phosphorus analysis. Phospholipid samples were dissolved in $200 \mu \mathrm{l}$ chloroform/methanol $(2: 1)$ and separated by two-dimensional chromatography on activated silica gel $G$ plates $(20 \times 10 \mathrm{~cm}$, Merck) according to Steiner \& Lester (1972). Phospholipids were visualized using the phosphomolybdate spray reagent (Dittmer \& Lester, 1964), which was prepared fresh every 6 months. The identity of each glycerophospholipid spot was determined by $(a)$ comparing the $R_{F}$ values of the sample spots with those of authentic standards (Sigma), and (b) determining their reactions with the dragendorf, ninhydrin, and ammoniacal silver nitrate reagents (Skidmore \& Entenmann, 1962). For quantitative measurements, spots were scraped from thin-layer chromatography (TLC) plates and the phosphorus contents determined using the methods of Rouser et al. (1970).

Inositol fractionation. The crude lipid extract prepared as described above was fractionated into three major classes of inositol-containing lipids according to the scheme in Fig. 1. The lipid extract was taken to dryness in vacuo at $45^{\circ} \mathrm{C}$ and non-polar lipids were extracted from the residue in $10 \mathrm{ml}$ acetone. The residue was then dissolved in $5 \mathrm{ml}$ chloroform/methanol (2:1) and the polar phosphosphingolipids and glycerophospholipids were separated on the basis of their differential stability to alkaline hydrolysis (Smith \& Lester, 1974).

\section{RESULTS}

\section{Effect of validamycin $A$ on the inositol content of Rhizoctonia cerealis and other fungi}

Validamycin $\mathrm{A}(1 \mu \mathrm{M})$ caused a $62 \%$ reduction in the inositol content of $R$. cerealis mycelia and also caused a significant reduction in the concentration of inositol in the culture supernatant: a $62 \%$ reduction in the unhydrolysed culture supernatant and a $50 \%$ reduction in the hydrolysed culture supernatant (Table 1). However, addition of the antibiotic to cultures of $R$. cerealis $6 \mathrm{~d}$ after inoculation (stationary phase) had no effect on either the endogenous or the exogenous inositol levels of the culture (Table 1). 
Table 1. Effect of validamycin $A$ on the distribution of inositol in $R$. cerealis cultures

$R$. cerealis was grown at $25^{\circ} \mathrm{C}$ in Vogel's medium containing $5 \mathrm{~mm}$-glucose in shake flask culture in the presence and absence of $1 \mu \mathrm{M}$-validamycin $\mathrm{A}$. Validamycin $\mathrm{A}$ was added at 0 or $6 \mathrm{~d}$ after inoculation and the mycelia and culture supernatant were harvested $8 \mathrm{~d}$ after inoculation. Mycelia and culture supernatant were hydrolysed in $6 \mathrm{M}-\mathrm{HCl}$ for $40-44 \mathrm{~h}$ and $20-25 \mathrm{~h}$ respectively and the inositol liberated was measured by bioassay. The inositol content of unhydrolysed culture supernatant was also determined by bioassay. The results are the means of five replicate cultures \pm SE.

\begin{tabular}{|c|c|c|c|}
\hline & \multicolumn{3}{|c|}{ Inositol content of cultures grown in: } \\
\hline & $\begin{array}{c}\text { Medium } \\
\text { lacking } \\
\text { validamycin A }\end{array}$ & $\begin{array}{c}\text { Medium } \\
\text { containing } 1 \mu \mathrm{M}- \\
\text { validamycin } \mathrm{A}\end{array}$ & $\begin{array}{c}\text { Medium to } \\
\text { which } 1 \mu \mathrm{M}- \\
\text { validamycin } \mathrm{A} \\
\text { was added } \\
6 \mathrm{~d} \text { after } \\
\text { inoculation }\end{array}$ \\
\hline Mycelium* & $2.51 \pm 0.32$ & $0.96 \pm 0.19 \ddagger$ & $2.71 \pm 0.36$ \\
\hline $\begin{array}{l}\text { Unhydrolysed culture } \\
\text { supernatant } \dagger\end{array}$ & $0.49 \pm 0.08$ & $0.12 \pm 0.01 \ddagger$ & $0.42 \pm 0.05$ \\
\hline $\begin{array}{l}\text { Hydrolysed culture } \\
\text { supernatant } \dagger\end{array}$ & $1 \cdot 71 \pm 0.22$ & $0.86 \pm 0.10 \ddagger$ & $1 \cdot 51 \pm 0.39$ \\
\hline
\end{tabular}

Table 2. Effect of validamycin $A$ on the inositol content of fungi 'morphologically' sensitive and insensitive to the antibiotic

Colonies were grown at $25^{\circ} \mathrm{C}$ on Vogel's medium or modified Vogel's medium containing 5 mm-glucose in the presence and absence of $25 \mu \mathrm{M}$-validamycin $\mathrm{A}$ in thin-layer liquid culture. Colonies were harvested $2 \mathrm{~d}$ after inoculation in the case of $N$. crassa and $5 \mathrm{~d}$ for the other fungi. The mycelium was hydrolysed in $6 \mathrm{M}-\mathrm{HCl}$ for $40-44 \mathrm{~h}$ and the inositol liberated was determined by bioassay. Results are expressed as $\mu \mathrm{g}$ inositol (mg mycelial dry weight) ${ }^{-1}$ and are the means of five replicates $\pm \mathrm{SE}$.

\begin{tabular}{|c|c|c|c|}
\hline \multirow[b]{2}{*}{ Organism } & \multirow[b]{2}{*}{$\begin{array}{l}\text { Sensitivity } \\
\text { to antibiotic* }\end{array}$} & \multicolumn{2}{|c|}{$\begin{array}{c}\text { Total inositol content }\left(\mu \mathrm{g} \mathrm{mg}^{-1}\right) \\
\text { of cultures grown on: }\end{array}$} \\
\hline & & $\begin{array}{c}\text { Medium } \\
\text { lacking } \\
\text { validamycin A }\end{array}$ & $\begin{array}{c}\text { Medium } \\
\text { containing } 25 \mu \mathrm{M}^{-} \\
\text {validamycin } \mathrm{A}\end{array}$ \\
\hline $\begin{array}{l}\text { Bjerkandera adusta } \\
\text { Pleurotus ostreatus } \\
\text { Neurospora crassa } \\
\text { Aspergillus nidulans }\end{array}$ & $\begin{array}{l}+ \\
+ \\
- \\
-\end{array}$ & $\begin{array}{r}4.5 \pm 0.4 \\
4.2 \pm 0.5 \\
8.3 \pm 1.2 \\
12.9 \pm 0.9\end{array}$ & $\begin{array}{l}1.3 \pm 0.1 \dagger \\
0.9 \pm<0.1 \dagger \\
8.7 \pm 0.8 \\
13.3 \pm 1.5\end{array}$ \\
\hline
\end{tabular}

* +, organism sensitive to antibiotic as indicated by increased hyphal branching in its presence; -, organism insensitive to antibiotic as indicated by unaltered hyphal branching in its presence.

$\dagger$ Significantly different ( $t$-test, $P<0 \cdot 05$ ) from control.

The effects of validamycin A on the inositol content of fungi sensitive (as indicated by increased hyphal branching in the presence of the antibiotic) and insensitive to the antibiotic are shown in Table 2. The inositol contents of Bjerkandera adusta and Pleurotus ostreatus, fungi sensitive to the antibiotic (Robson et al., 1988), were reduced by validamycin A by $71 \%$ and $79 \%$ respectively. However, the inositol contents of Neurospora crassa and Aspergillus nidulans, fungi insensitive to the antibiotic (Robson et al., 1988), were not significantly affected by the antibiotic. 


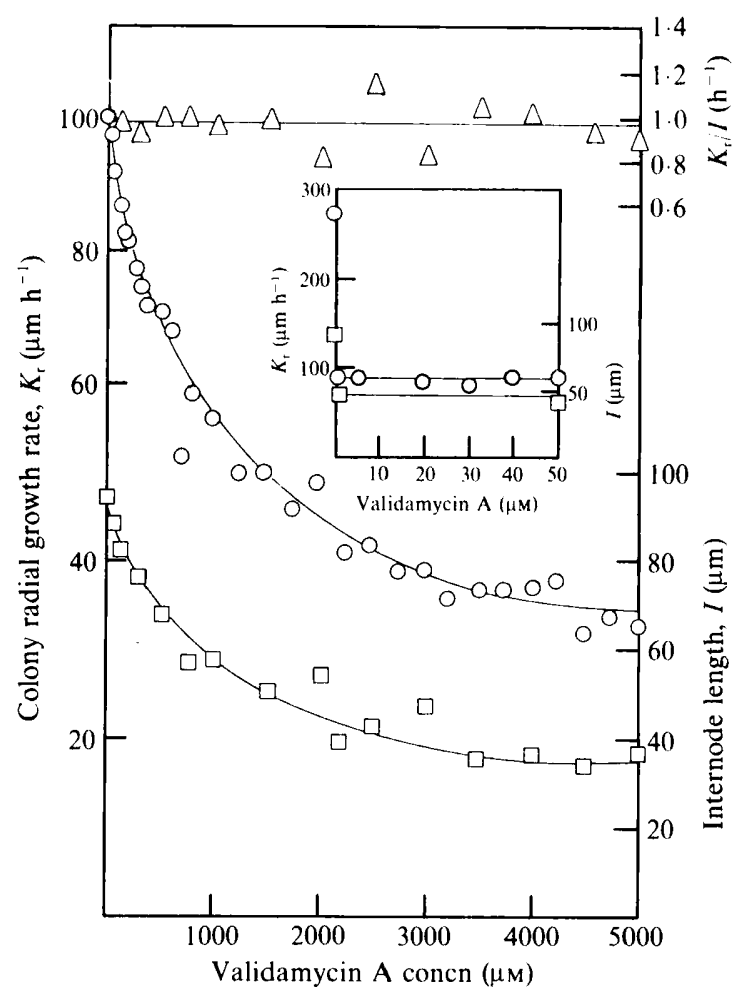

Fig. 2. Effect of validamycin A on the colony radial growth rate, $K_{\mathrm{r}}(\mathrm{O})$, and internode length, $I(\square)$, of $R$. cerealis. Colonies were grown at $25^{\circ} \mathrm{C}$ on Vogel's medium containing $5 \mathrm{~mm}$-glucose in the presence and absence of various concentrations of validamycin A. All colony radial growth rates were measured during the linear growth phase and two measurements were made at right-angles on five replicate colonies. Each internode length was the mean of 45 internode lengths taken from the first three consecutive internodes in each of three leading hyphae from the margins of five replicate colonies which were in the linear phase of growth. The inset represents the effect of validamycin $A$ up to a concentration of $50 \mu \mathrm{M} . \triangle$, Ratio $K_{\mathrm{r}} / I$.

Effect of validamycin $A$ on the colony radial growth rate, internode length and inositol content of $R$. cerealis

Increasing the antibiotic concentration in the medium from 1 to $5000 \mu \mathrm{M}$ caused an exponential decline in both the colony radial growth rate $\left(K_{\mathrm{r}}\right)$ and internode length $(I)$ of $R$. cerealis (Fig. 2), but had no appreciable effect on the ratio $\left(K_{\mathrm{r}} / I\right)$ between these two parameters. Validamycin A concentrations between 1 and $10 \mu \mathrm{M}$ caused an approximately $50 \%$ decrease in the inositol content of $R$. cerealis colonies (inset to Fig. 3). Further decreases in inositol content were observed at validamycin A concentrations between 20 and $2000 \mu \mathrm{M}$ (Fig. 3); at validamycin A concentrations of $2 \mathrm{~mm}$ and above, antibiotic-treated colonies contained only about $17 \%$ of the concentration of inositol observed in control colonies. Positive correlations were found between inositol content of the biomass and colony radial growth rate $(r=0.87)$, and between inositol content of the biomass and internode length $(r=0.95)$ (Fig. 4). However, for colony radial growth rate and inositol content, the relationship was only maintained up to $1.40 \mu \mathrm{g}$ inositol (mg fungal dry weight $)^{-1}$.

\section{Effect of validamycin $A$ on the distribution of inositol in $R$. cerealis}

Inositol present in $R$. cerealis was fractionated according to the scheme in Fig. 1 . Only $77 \%$ of the total inositol present in untreated (control) mycelia was removed by the extraction procedure, the remainder being cell bound. The alkali-deacylatable fraction (F3), the alkali- 


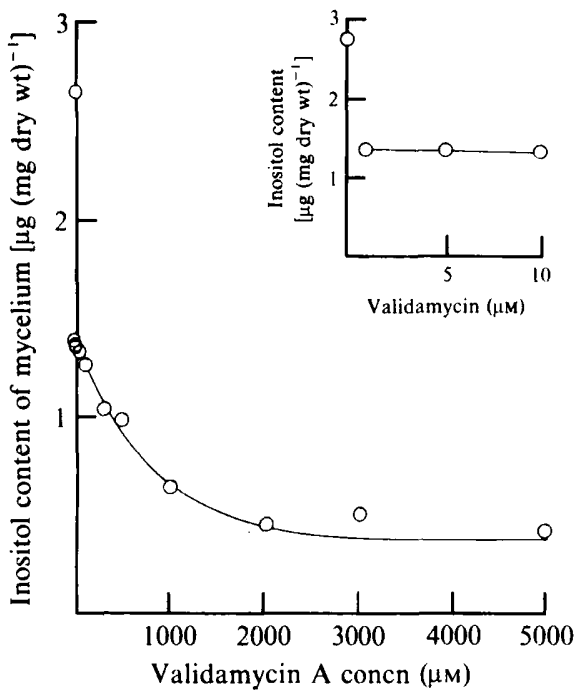

Fig. 3

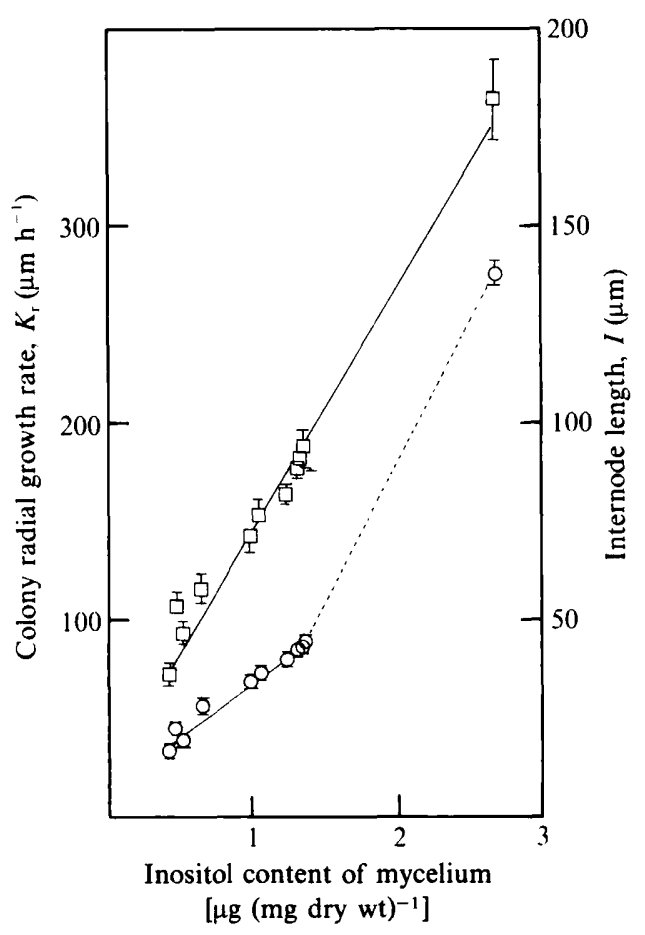

Fig. 4

Fig. 3. Effect of validamycin A on the inositol content of $R$. cerealis. Colonies were grown at $25^{\circ} \mathrm{C}$ on Vogel's agar medium containing $5 \mathrm{mM}$-glucose in the presence and absence of various concentrations of validamycin A. Colonies were harvested from the Cellophane overlays $5 \mathrm{~d}$ after inoculation. Mycelium was hydrolysed in $6 \mathrm{M}-\mathrm{HCl}$ for $40-44 \mathrm{~h}$ and the inositol liberated was determined by bioassay. Results are expressed as $\mu \mathrm{g}$ inositol (mg mycelial dry weight) ${ }^{-1}$ and represent the means of two determinations made on each of five replicate colonies. The inset represents the effect of validamycin A concentrations of $10 \mu \mathrm{M}$ and below on inositol content.

Fig. 4. Relationships between inositol content and radial growth rate $(O)$ and between inositol content and internode length $(\square)$ of colonies of $R$. cerealis. The inositol content of $R$. cerealis colonies from Fig. 3 was plotted against the corresponding colony radial growth rates and internode lengths from Fig. 2, $\pm \mathrm{SE}$. Lines were plotted using least-squares regression analysis.

stable fraction (F4), and the acetone-soluble fraction (F2) accounted for 31,58 and $11 \%$ of the total extractable inositol, respectively (Table 3). Validamycin A caused significant decreases in the inositol content of all except the acetone-soluble fraction; the total inositol content of the mycelium was reduced by $47 \%$ and decreases of 52,57 and $40 \%$ were observed for the nonextractable fraction (F1), the alkali-deacylatable fraction (F3) and the alkali-stable fraction (F4) respectively (Table 3). Analysis of the effect of validamycin A on the phospholipid composition of $R$. cerealis revealed that, compared to control cultures, the antibiotic caused a significant decrease $(53 \%)$ in the phosphatidylinositol concentration and significant increases in the levels of phosphatidylserine ( $23 \%$ ) and an unknown, non-inositol-containing phospholipid, unknown B $(162 \%)$ (Table 4$)$. Other phospholipids and the overall phospholipid content of the mycelium were not significantly affected by the antibiotic.

Effects of validamycin $A$ on the inositol content of fungi sensitive and insensitive to the antibiotic

Validamycin A mainly affects fungi belonging to the Basidiomycotina (Robson et al., 1988) and it is possible that sensitivity to the antibiotic is dependent upon the inositol content of the biomass, i.e. fungi which have a high inositol content may be more sensitive to validamycin $A$ 
Table 3. Distribution of inositol in $R$. cerealis grown in the presence and absence of validamycin $A$

$R$. cerealis was grown in shake flask culture for $4 \mathrm{~d}$ at $25^{\circ} \mathrm{C}$ in Vogel's medium containing 5 mm-glucose in the presence and absence of $1 \mu \mathrm{M}$-validamycin $\mathrm{A}$. The mycelium was then fractionated and each fraction hydrolysed in $6 \mathrm{M}-\mathrm{HCl}$ for $40-44 \mathrm{~h}$. The inositol liberated was measured by bioassay. Results are expressed as $\mu \mathrm{g}$ inositol (mg mycelial dry weight) ${ }^{-1}$ and represent the means of two determinations on 10 replicate cultures \pm SE.

\begin{tabular}{lcc} 
Fraction & $\begin{array}{c}\text { Medium lacking } \\
\text { validamycin A }\end{array}$ & $\begin{array}{c}\text { Inositol content }\left(\mu \mathrm{g} \mathrm{mg}^{-1}\right) \\
\text { of cultures grown in: }\end{array}$ \\
\cline { 2 - 3 } & $0.51 \pm 0.05$ & $0.23 \pm 0.02^{*}$ \\
ll fraction, F1 & $0.11 \pm 0.02$ & $0.06 \pm 0.03$ \\
soluble fraction, F2 & $0.30 \pm 0.01$ & $0.13 \pm 0.01^{*}$ \\
table fraction, F4 & $0.57 \pm 0.07$ & $0.35 \pm 0.04^{*}$ \\
mycelium & $1.57 \pm 0.06$ & $0.84 \pm 0.08^{*}$ \\
age inositol recovered & 95 & 91
\end{tabular}

* Significantly different $(t$-test, $P<0.05)$ from control.

Table 4. Effect of validamycin $A$ on the glycerophospholipid composition of $R$. cerealis

$R$. cerealis was grown at $25^{\circ} \mathrm{C}$ in shake flask culture in Vogel's medium containing $5 \mathrm{~m}$ M-glucose in the presence and absence of $1 \mu \mathrm{M}$-validamycin $\mathrm{A}$. Cultures were harvested $4 \mathrm{~d}$ after inoculation and extracted phospholipids were separated by two-dimensional TLC and quantified by phosphorus analysis. Results are expressed as $\mu \mathrm{mol}$ phosphorus ( $\mathrm{g}$ mycelial dry weight) ${ }^{-1}$ and represent the means of eight replicate cultures from two independent experiments \pm SE.

\begin{tabular}{|c|c|c|}
\hline \multirow[b]{2}{*}{ Phospholipid } & \multicolumn{2}{|c|}{$\begin{array}{c}\text { Phospholipid composition }\left(\mu \mathrm{mol} \mathrm{P} \mathrm{g}^{-1}\right) \\
\text { of cultures grown in: }\end{array}$} \\
\hline & $\begin{array}{l}\text { Medium lacking } \\
\text { validamycin } \mathrm{A}\end{array}$ & $\begin{array}{l}\text { Medium containing } \\
1 \mu \mathrm{M} \text {-validamycin } \mathrm{A}\end{array}$ \\
\hline Phosphatidic acid & $0.29 \pm 0.09$ & $0.27 \pm 0.08$ \\
\hline Phosphatidylinositol & $2.09 \pm 0.14$ & $0.98 \pm 0.13^{*}$ \\
\hline Phosphatidylserine & $3.68 \pm 0.59$ & $4.73 \pm 0.27^{*}$ \\
\hline Phosphatidylcholine & $19.82 \pm 1.34$ & $18.63 \pm 1.57$ \\
\hline Phosphatidylethanolamine & $10.93 \pm 0.84$ & $9.90 \pm 0.69$ \\
\hline Phosphatidylglycerol & $0.35 \pm 0.14$ & $0.39 \pm 0.17$ \\
\hline Diphosphatidylglycerol & $1.78 \pm 0.10$ & $1.51 \pm 0.05$ \\
\hline Unknown A & $0.13 \pm 0.07$ & $0.11 \pm 0.04$ \\
\hline Unknown B & $0.13 \pm 0.03$ & $0.34 \pm 0.05^{*}$ \\
\hline Total applied & $42.69 \pm 3.21$ & $40.44 \pm 4.54$ \\
\hline Percentage $P$ recovered & 91 & 90 \\
\hline
\end{tabular}

* Significantly different ( $t$-test, $P<0.05$ ) from control.

than fungi which have a low inositol content. A survey was therefore made of the inositol contents of fungi sensitive and insensitive to validamycin A (Table 5).

The inositol contents of validamycin A sensitive fungi ranged from $1.7 \mu \mathrm{g}$ (mg dry weight $)^{-1}$ for Chaetomium globosum to $7.0 \mu \mathrm{g}$ (mg dry weight $)^{-1}$ for Heterobasidion annosum. For validamycin $\mathrm{A}$ insensitive fungi the inositol contents ranged from $0.4 \mu \mathrm{g}(\mathrm{mg} \text { dry wt })^{-1}$ for Cladosporium cucumeris to $14.6 \mu \mathrm{g}$ (mg dry weight $)^{-1}$ for Aspergillus nidularis. Thus, there was no obvious relationship between inositol content of a fungus and its sensitivity to the antibiotic. 
Table 5. Inositol content of validamycin $A$ sensitive (indicated by increased branch frequency in the presence of the antibiotic) and validamycin $A$ insensitive (indicated by unchanged morphology) fungi

Fungi were grown at $25^{\circ} \mathrm{C}$ in $10 \mathrm{ml}$ Vogel's or modified Vogel's liquid medium containing $50 \mathrm{~mm}$ glucose. The stationary liquid cultures were harvested when the concentration of glucose remaining in the medium was less than $10 \mathrm{~mm}$. The mycelium was hydrolysed in $6 \mathrm{M}-\mathrm{HCl}$ for $40-44 \mathrm{~h}$ and the inositol liberated was measured by bioassay. Results are expressed as $\mu \mathrm{g}$ inositol (mg mycelial dry weight $)^{-1}$ and represent the means of five replicate cultures \pm SE.

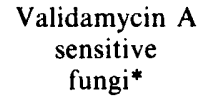

Heterobasidion annosum

Cystoderma amianthinum

Agaricus silvicola

Flammulina velutipes

Pseudotrametes gibbosa

Bjerkandera adusta

Phlebia rufa

Fusarium culmorum ${ }^{\mathrm{D}}$

Phlebia radiata

Pleurotus ostreatus

Daedalea quercina

Rhizoctonia cerealis ${ }^{D}$

Lentinus edodes

Chaetomium globosum ${ }^{\mathrm{A}}$

Total inositol
content of the
biomass $\left(\mu \mathrm{g} \mathrm{m}^{-1}\right)$

$$
\begin{aligned}
& 7.0 \pm 0.2 \\
& 6.5 \pm 0.8 \\
& 6.4 \pm 0.5 \\
& 6.2 \pm 0.5 \\
& 5.9 \pm 0.4 \\
& 5.3 \pm 0.6 \\
& 4.5 \pm 0.4 \\
& 4.4 \pm 0.2 \\
& 4.2 \pm 0.5 \\
& 4.0 \pm 0.3 \\
& 3.6 \pm 0.2 \\
& 2.7 \pm 0.4 \\
& 2.0 \pm 0.2 \\
& 1.7 \pm 0.1
\end{aligned}
$$

Validamycin A
insensitive
fungi

Aspergillus nidulans

Penicillium chrysogenum ${ }^{\mathrm{D}}$

Botrytis fabae ${ }^{\mathrm{D}}$

Neurospora crassa

Sordaria fimicola

Pythium ultimum ${ }^{\circ}$

Sporotrichum carnis

Saprolegnia ferax ${ }^{\mathrm{O}}$

Chocliobolus sativus

Paecilomyces farinosus

Zygorhyncus nidlii ${ }^{\mathrm{M}}$

Mycotypha africana ${ }^{\mathrm{M}}$

\begin{tabular}{|c|c|c|}
\hline \multirow[b]{2}{*}{$\begin{array}{l}\text { Inositol concn. } \\
\text { in medium }(\mathrm{mM})\end{array}$} & \multicolumn{2}{|c|}{$\begin{array}{c}\text { Colony radial growth rate, } K_{\mathrm{r}}\left(\mu \mathrm{m} \mathrm{h}^{-1}\right) \\
\text { of cultures grown on: }\end{array}$} \\
\hline & $\begin{array}{l}\text { Medium lacking } \\
\text { validamycin } \mathrm{A}\end{array}$ & $\begin{array}{l}\text { Medium containing } \\
1 \mu \mathrm{M} \text {-validamycin } \mathrm{A}\end{array}$ \\
\hline 0 & $247 \pm 3$ & $61 \pm 1$ \\
\hline 0.01 & $252 \pm 6$ & $61 \pm<1$ \\
\hline $0 \cdot 1$ & $245 \pm 4$ & $66 \pm 2$ \\
\hline 1.0 & $249 \pm 7$ & $73 \pm 3 \ddagger$ \\
\hline $5 \cdot 0$ & $250 \pm 11$ & $79 \pm 3 \ddagger$ \\
\hline $10 \cdot 0$ & $127 \pm 2+$ & $33 \pm<1 \ddagger$ \\
\hline
\end{tabular}

Cladosporium cucumeris
Total inositol content of the biomass $\left(\mu \mathrm{g} \mathrm{mg}^{-1}\right)$

* Basidiomycotina unless otherwise indicated (A, Ascomycotina; D, Deuteromycotina).

$\dagger$ Ascomycotina unless otherwise indicated (O, Oomycetes; M, Mucorales; D, Deuteromycotina).

Table 6. Partial reversal of the effect of validamycin $A$ on colony radial growth rate of $R$. cerealis by inositol

$R$. cerealis colonies were grown at $25^{\circ} \mathrm{C}$ on plates of Vogel's medium plus $50 \mathrm{~mm}$-mannitol, in the presence and absence of $1 \mu \mathrm{M}$-validamycin $\mathrm{A}$ and containing various inositol concentrations. All colony radial growth rates were measured during the linear growth phase. Two measurements were made at right angles on five replicate plates at various time intervals to give 10 colony radial growth rates which were treated as independent replicates \pm SE.

* Each result significantly different $(t$-test, $P<0.05)$ from control lacking validamycin A.

$\dagger$ Significantly different $(t$-test, $P<0.05)$ from control lacking inositol.

$\ddagger$ Significantly different $(t$-test, $P<0.05)$ from control lacking inositol.

\section{Partial reversal of the effects of validamycin $A$ by inositol}

The effects of inositol concentration on the radial growth rates of control and validamycin $A$ treated colonies grown on medium containing $50 \mathrm{~mm}$-mannitol as the carbon source are shown in Table 6; mannitol is a poor carbon source for growth and was used in this experiment because glucose represses inositol uptake (G. D. Robson, P. J. Kuhn \& A. P. J. Trinci, unpublished 
Table 7. Effect of inositol on the phosphatidylinositol content of untreated and validamycin $A$ treated $R$. cerealis

\begin{abstract}
$R$. cerealis was grown at $25^{\circ} \mathrm{C}$ in shake flask culture in Vogel's medium containing $50 \mathrm{~mm}$-mannitol in the presence and absence of $1 \mu \mathrm{M}$-validamycin A and $5 \mathrm{~mm}$-inositol. Mycelium was harvested after $5 \mathrm{~d}$, during the late exponential phase and extracted phospholipids were separated by two-dimensional TLC. Phosphatidylinositol was quantified by phosphorus analysis. Results are expressed as $\mu$ mol phosphorus ( $\mathrm{g}$ mycelial dry weight $)^{-1}$ and represent the means of five replicate flasks $\pm \mathbf{S E}$.
\end{abstract}

\begin{tabular}{|c|c|c|}
\hline \multirow[b]{2}{*}{$\begin{array}{l}\text { Concn of inositol in } \\
\text { medium (mM) }\end{array}$} & \multicolumn{2}{|c|}{$\begin{array}{l}\text { Phosphatidylinositol content }\left(\mu \mathrm{mol} \mathrm{P} \mathrm{g}^{-1}\right) \\
\text { of cultures grown in: }\end{array}$} \\
\hline & $\begin{array}{l}\text { Medium lacking } \\
\text { validamycin } \mathrm{A}\end{array}$ & $\begin{array}{l}\text { Medium containing } \\
1 \mu \mathrm{M} \text {-validamycin } \mathrm{A}\end{array}$ \\
\hline 0 & $2.72 \pm 0.22$ & $1.20 \pm 0.17^{*}$ \\
\hline 5 & $3.05 \pm 0.18 \dagger$ & $1 \cdot 16 \pm 0 \cdot 10^{*} \dagger$ \\
\hline
\end{tabular}

observation). In the absence of validamycin A, concentrations of inositol up to $5 \mathrm{~mm}$ had no significant effect on colony radial growth rate. However, colony radial growth rate was significantly decreased in the presence of $10 \mathrm{~mm}$ inositol. In the presence of validamycin $\mathrm{A}, 1$ and $5 \mathrm{~mm}$ inositol significantly increased colony radial growth rate compared to the validamycin A control (colonies grown on medium containing validamycin A but lacking inositol) by $20 \%$ and $30 \%$ respectively, but $10 \mathrm{~mm}$-inositol caused a significant decrease in colony radial growth rate. Measurements of the phosphatidylinositol content of $R$. cerealis grown on medium containing $50 \mathrm{mM}$-mannitol as the carbon source revealed that $5 \mathrm{~mm}$-inositol had no significant effect on the endogenous phosphatidylinositol levels of validamycin A inhibited mycelia (Table 7).

\title{
DISCUSSION
}

Validamycin A caused reductions in the concentrations of endogenous and exogenous inositol in cultures of $R$. cerealis but did not appear to stimulate the degradation of inositol-containing compounds formed prior to exposure of the fungus to the antibiotic (Table 1). Validamycin $A$ treated colonies of Bjerkandera adusta and Pleurotus ostreatus (fungi which are sensitive to the antibiotic as judged by increased hyphal branching) also had a reduced inositol content compared to control colonies, whereas the inositol contents of Neurospora crassa and Aspergillus nidulans (fungi which are insensitive to the antibiotic) were unaffected by validamycin A (Table 2). Over the concentration range 1 to $5000 \mu \mathrm{M}$, the internode length and the radial growth rate of colonies of $R$. cerealis decreased with the log of the concentration of the antibiotic in the medium (Fig. 2). However, although the inositol content of colonies decreased with increase in validamycin A concentration over the range 1 to $2000 \mu \mathrm{M}$, the inositol contents of colonies grown on 1 and $10 \mu \mathrm{M}$ validamycin A were not significantly different (Fig. 3). The ratio $K_{\mathrm{r}} / I$ remained approximately constant over the range of antibiotic concentrations tested (Fig. 2). Internode length is an indicator of hyphal branch frequency and therefore the present result is consistent with the hypothesis of Morrison \& Righelato (1974) and Jejelowo \& Trinci (1988) that hyphal growth unit length (another indicator of hyphal branch frequency) is a determinant of peripheral growth zone width (Trinci, 1971) and hence of colony radial growth rate. Thus, the reduction in the radial growth rate of validamycin A treated colonies results from the effect of the antibiotic on hyphal branching.

Positive linear correlations were observed in $R$. cerealis between inositol content of the biomass and colony radial growth rate, and between inositol content of the biomass and the frequency of hyphal branching (internode length) (Fig. 4). Previous studies with inositol auxotrophs of $N$. crassa have also shown that decreased levels of endogenous inositol are 
associated with the formation of highly branched mycelia which form colonies that expand at much slower rates than control colonies (Fuller \& Tatum, 1956; Shatkin \& Tatum, 1961; Hanson \& Brody, 1979).

In fungi, as in other eukaryotes, inositol is present primarily in membranes, principally in the form of glycerophospholipids and phosphosphingolipids (Brennan \& Losel, 1978). In R. cerealis, $66 \%$ of the total inositol was extracted in the lipid fraction (Table 3) and the remainder was probably present as inositol-containing lipid tightly associated with the cell wall (Hanson \& Brody, 1979). The inositol extracted from the biomass was separated into three fractions representing inositol-containing phospholipid (31\% of the total), phosphosphingolipid $(58 \%)$ and glycolipid (11\%). Validamycin A caused a $57 \%$ decrease in the level of inositol-containing phospholipid but a smaller reduction in the level of inositol-containing phosphosphingolipid $(39 \%)$. Phosphatidylinositol (PI) was the only detectable inositol-containing phospholipid in $R$. cerealis although two minor phosphorylated derivatives have been detected in Saccharomyces cerevisiae (Lester \& Steiner, 1968). Validamycin A caused a $47 \%$ reduction in PI content which was correlated with a rise in the levels of phosphatidylserine (PS) and an unidentified phospholipid (unknown B, Table 4). Other phospholipids in $R$. cerealis were not significantly affected by the validamycin $A$ treatment and the total phospholipid content of the fungus was unaffected by the antibiotic. A rise in PS associated with a decline in PI has also been reported for inositol auxotrophs of $N$. crassa grown on inositol-deficient medium and is thought to reflect a regulatory mechanism which serves to conserve membrane charge by maintaining a constant ratio between anionic and zwitterionic phospholipids in the membrane (Hubbard \& Brody, 1975).

Inositol auxotrophs of $N$. crassa and $S$. cerevisiae grown on medium containing sub-optimal concentrations of inositol have been correlated with changes in cell wall composition (Hanson \& Brody, 1979; Hanson \& Lester, 1982), and studies with colonial (highly branched) mutants of $N$. crassa have also demonstrated a correlation between changes in cell wall composition and the formation of a highly branched mycelium. The activities of some membrane-bound enzymes display a requirement for a particular phospholipid; in fungi, for example, maximal in vitro activities of chitin synthase (Duran \& Cabib, 1978; Vermeulen \& Wessels, 1983; Montgomery \& Gooday, 1985) and chitinase (Humphreys \& Gooday, 1984) are dependent on the presence of phosphatidylcholine (PC). The organophosphorus fungicides ediphenphos (Hinosan) and IBP (Kitazin) reduce the levels of PC in Pyricularia oryzae by inhibiting $S$-adenosylmethionine transferase, and, apparently, this change in membrane composition results in a decrease in the rate of chitin biosynthesis (Kodama et al., 1979, 1980; Yoshida et al., 1984). Ediphenphos also reduces PC levels in Fusarium graminearum, and this is associated with an increase in hyphal branching (M. Wiebe, G. D. Robson \& A. P. J. Trinci, unpublished results). A reduction in the levels of inositol-containing lipids in the membrane may therefore influence the activity of membrane-bound enzymes, including those involved in wall synthesis and assembly. Similarly, the paramorphogen L-sorbose is thought to stimulate branching in $N$. crassa by inhibiting the activity of $\beta$-1,3-glucan synthase (Mishra \& Tatum, 1972), and other paramorphogens may also be found to produce their effects through alterations in wall biosynthetic enzymes. In an inositol auxotroph of $S$. cerevisiae, the activity of the membrane-bound enzyme UDP- $N$-acetylglucosamine-dolichol phosphate $N$-acetylglucosamine-1-phosphotransferase, which is involved in mannan synthesis, requires PI for maximal activity and is inhibited by an increase in the ratio between inositol-containing phosphosphingolipid and PI (Hanson \& Lester, 1982; Hanson, 1984). When this mutant is grown at sub-optimal concentrations of inositol there is a decrease in the level of PI, a smaller decrease in the levels of inositol-containing phosphosphingolipid (increasing the ratio between the two) and a reduction in the mannan content of the cell wall. In view of this result, it is significant that validamycin A causes a decrease in the $\beta-1,3-$ glucomannan fraction of the cell wall of $R$. solani (Kido et al., 1986).

In fungal mycelia there is a relationship between branch frequency, hyphal extension and specific growth rate $(\mu)$ (Trinci, 1974, 1984): $G=E / \mu$, where $G$ is the hyphal growth unit length (mean length of hypha associated with each tip; Trinci, 1974) and $E$ is the mean rate of extension of the hyphae in the mycelium. Hyphal extension involves the incorporation of new wall 
material at the hyphal tip and this is rapidly followed by the formation of linkages between the newly formed polymers which results in wall rigidification (Wessels et al., 1983; Sonnenberg et al., 1985). A decrease in the maximum activity of an enzyme(s) involved in the biosynthesis or assembly of the tip wall may lead to a decrease in hyphal extension rate but this need not necessarily be correlated with a decrease in specific growth rate. Any factor which reduces hyphal extension rate without affecting specific growth rate will lead to a reduction in the hyphal growth unit, i.e. an increase in branch frequency (Trinci, 1984). This increase in branch frequency will, in turn, result in a decrease in colony radial growth rate (Jejelowo \& Trinci, 1988).

Inositol is synthesized from glucose-6-phosphate, first with the formation of inositol 1phosphate (catalysed by the enzyme inositol-1-phosphate synthase) which is then dephosphorylated by inositol-1-phosphatase to form inositol (Chen \& Charalampous, 1966; Aradi et al., 1982; Escamilla et al., 1982). Addition of inositol to medium containing validamycin $\dot{A}$ and mannitol caused only a slight increase in the colony radial growth rate (Table 6) and had no significant effect on the PI content of the biomass (Table 7); this suggests that the antibiotic does not inhibit inositol biosynthesis directly, as its effects cannot be antagonized by exogenous inositol. However, validamycin A may inhibit the incorporation of inositol into PI, a reaction catalysed by the membrane-bound enzyme PI synthase (Steiner \& Lester, 1972; Fischl \& Carman, 1983). Inhibition at this point would lead to a rise in the internal pool of inositol, which may inhibit inositol biosynthesis by feedback inhibition. Inositol-1-phosphate synthase is strongly inhibited by inositol in vitro (Culbertson et al., 1976; Zsindeley et al., 1983).

The present work with validamycin A suggests that phospholipid biosynthesis is a target for fungicides which display selective toxicity.

We thank the Science and Engineering Research Council for a CASE studentship to the University of Manchester and Shell Research Limited, Sittingbourne.

\section{REFERENCES}

ANGUS, W. W. \& Lester, R. L. (1972). Turnover of inositol and phosphorus-containing lipids in Saccharomyces cerevisiae; extracellular accumulation of glycerophosphorylinositol derived from phosphatidylinositol. Archives of Biochemistry and Biophysics 151, 483-495.

Aradi, J., Zsindeley, A., Kiss, A., Szabolcs, M. \& SCHABliK, M. (1982). Separation of Neurospora crassa myo-inositol-1-phosphate synthase from glucose-6-phosphate dehydrogenase by affinity chromatography. Analytical Biochemistry 12, 137-151.

Brennan, P. J. \& Losel, D. M. (1978). Physiology of fungal lipids: selected topics. Advances in Microbial Physiology 17, 47-179.

Chen, I. W. \& Charalampous, F. C. (1966). Biochemical studies on inositol. X. Partial purification of yeast inositol-1-phosphatase and the separation from glucose-6-phosphate cyclase. Archives of Biochemistry and Biophysics 117, 154-157.

Culbertson, M. R., Donahue, T. F. \& Henry, S. A. (1976). Control of inositol biosynthesis in Saccharomyces cerevisiae: 1. Properties of a repressible enzyme system in extracts of wild type (ino ${ }^{+}$) cells. Journal of Bacteriology 126, 232-236.

DitTMer, J. C. \& Lester, R. L. (1964). A simple specific spray reagent for the detection of phospholipids on thin-layer chromatograms. Journal of Lipid Research 5, 126-127.

Duran, A. \& CABIB, E. (1978). Solubilization and partial purification of yeast chitin synthetase. Conformation of the zymogenic nature of the enzyme. Journal of Biological Chemistry 253, 4419-4425.
Escamilla, J. E., Conteras, M., Martinez, A. \& Zentella-Piña, M. (1982). L-myo-inositol-1-phosphate synthase from Neurospora crassa: purification to homogeneity and partial characterisation. $A r$ chives of Biochemistry and Biophysics 218, 275-285.

Fischl, A. S. \& Carman, G. M. (1983). Phosphatidylinositol biosynthesis in Saccharomyces cerevisiae. Purification and properties of microsome-associated phosphatidylinositol synthase. Journal of Bacteriology 154, 304-311.

Fuller, R. C. \& TAtum, E. L. (1956). Inositol phospholipid in Neurospora crassa and its relationship to morphology. American Journal of Botany 43, $361-365$.

Hanson, B. A. (1984). Role of inositol-containing sphingolipids in Saccharomyces cerevisiae during inositol starvation. Journal of Bacteriology 159, 837842.

Hanson, B. A. \& Brody, S. (1979). Lipid and cell wall changes in an inositol-requiring mutant of Neurospora crassa. Journal of Bacteriology 138, 461-466.

HANSON, B. A. \& LeSTER, R. L. (1982). Effect of inositol starvation on the in vitro synthesis of mannan and $\mathrm{N}$-acetylglucosaminylpyrophosphoryldolichol in Saccharomyces cerevisiae. Journal of Bacteriology 151, 334-342.

Hubbard, S. C. \& Brody, S. (1975). Glycerophospholipid variation in choline and inositol auxotrophs of Neurospora crassa. Journal of Biological Chemistry 250, 7173-7178.

Humphreys, A. M. \& GOOdAY, G. W. (1984). Phospholipid requirement of microsomal chitinase 
from Mucor mucedo. Current Microbiology 11, 187190.

Jejelowo, O. \& TrincI, A. P. J. (1988). Effect of the paramorphogens, 3-O-methyl-D-glucose, glucosamine and L-sorbose on growth and morphology of Botrytis fabae. Transactions of the British Mycological Society 91, 653-660.

Kido, Y., Nagasato, T., Ono, K., Fujimoto, Y., UYEDA, M. \& Shibata, M. (1986). Changes in a cellwall component of Rhizoctonia solani inhibited by validamycin. Journal of Antibiotics 50, 1519-1525.

Kodama, O., Yamada, H. \& AKatsuka, J. (1979). Kitazin P, inhibitor of phosphatidylcholine biosynthesis in Pyricularia oryzae. Agricultural and Biological Chemistry 43, 1719-1725.

Kodama, O., Yamashita, K. \& Akatsuka, J. (1980). Hinosan, inhibitor of phosphatidylcholine biosynthesis in Pyricularia oryzae. Agricultural and Biological Chemistry 44, 1015-1021.

LESTER, R. L. \& STEINER, M. R. (1968). The occurrence of diphosphoinositide and triphosphoinositide in Saccharomyces cerevisiae. Journal of Biological Chemistry 243, 4889-4893.

Mishra, N. \& Tatum, E. (1972). The effect of Lsorbose on the polysaccharide synthetases of Neurospora crass.a. Proceedings of the National Academy of Sciences of the United States of America 69, 313-317.

Montgomery, G. W. G. \& Gooday, G. W. (1985). Phospholipid-enzyme interactions of chitin synthase of Coprinus cinereus. FEMS Microbiology Letters 27, 29-33.

Morrison, K. B. \& Righelato, R. C. (1974). The relationship between hyphal branching, specific growth rate and colony radial growth rate in Penicillium chrysogenum. Journal of General Microbiology 81, 517-520.

NioH, T. \& Mizushima, S. (1974). Effect of validamycin A on the growth and morphology of Pellicularia sasakii. Journal of General and Applied Microbiology 20, 373-383.

Paranjapye, V. N., Deshusses, J. \& Posternack, T. H. (1964). Sur une méthode simplifiée de dosage microbiologique du MS-inositol. Analytical chimica acta 31, 480-483.

Robson, G. D., Bell, S. D., Kuhn, P. J. \& Trincl, A. P. J. (1987). Glucose and penicillin concentrations in agar medium below fungal colonies. Journal of General Microbiology 133, 361-367.

Robson, G. D., Kuhn, P. J. \& Trinci, A. P. J. (1988). Effects of validamycin A on the morphology, growth and sporulation of Rhizoctonia cerealis, Fusarium culmorum and other fungi. Journal of General Microbiology 134, 3187-3194.

Rouser, G., Fleischer, S. \& Yamamoto, A. (1970). Two-dimensional thin-layer chromatographic separation of polar lipids and determination of phospholipids of phosphorus analysis of spots. Lipids 5 , 494-496.

Shatkin, A. J. \& Tatum, E. L. (1961). The relationship of m-inositol to morphology in Neurospora crassa. American Journal of Botany 48, 760-761.

Shibata, M., UYEDA, M. \& MORI, K. (1980). Reversal of validamycin inhibition by the hyphal extract of Rhizoctonia solani. Journal of Antibiotics 33, 679-681. Shibata, M., Uyeda, M. \& Mori, K. (1981). Stimula- tion of the extension of validamycin-inhibited hyphae by the hyphal extension factor present in Rhizoctonia solani. Agricultural and Biological Chemistry 44, 2241-2243.

SkidmoRe, W. D. \& EnTEnmann, C. (1962). Twodimensional thin layer chromatography of rat liver phosphatides. Journal of Lipid Research 3, 471-475.

SMITH, S. W. \& Lester, R. L. (1974). Inositol phosphorylceramide, a novel substance and chief rnember of a major group of yeast sphingolipids containing a single inositol phosphate. Journal of Biological Chemistry 249, 3395-3405.

Sonnenberg, A. S. M., Sietsma, J. H. \& Wessels, J. G. H. (1985). Spatial and temporal differences in the synthesis of $(1 \rightarrow 3) \beta$ and $(1 \rightarrow 6) \beta$ linkages in a cell wall glucan of Schizophyllum commune. Experimental Mycology 9, 3395-3405.

STEINER, M. R. \& LESTER, R. L. (1972). In vitro studies of phospholipid biosynthesis in Saccharomyces cerevisiae. Biochimica et biophysica acta 260 , 222-243.

Tatum, E. L., Barratt, R. W. \& Cutter, V. M (1949). Chemical induction of colonial paramorphs of Neurospora and Syncephalastrum. Science 109, 509-511.

TRINCI, A. P. J. (1971). Influence of peripheral growth zone on the radial growth rate of fungal colonies. Journal of General Microbiology 67, 325-344.

Trinci, A. P. J. (1974). A study of the kinetics of hyphal extension and branch initiation of fungal mycelia. Journal of General Microbiology 81, 225236.

TrincI, A. P. J. (1984). Antifungal agents which affect hyphal branching. In Mode of Action of Antifungal Agents, pp. 113-134. Edited by A. P. J. Trinci \& J. F. Ryley. Cambridge: Cambridge University Press.

TRINCI, A. P. J. (1985). Effect of validamycin A and Lsorbose on the growth and morphology of Rhizoctonia cerealis and Rhizoctonia solani. Experimental Mycology 9, 20-27.

Vermuelen, C. A. \& Wessels, J. G. H. (1983). Evidence for a phospholipid requirement of chitin synthase in Schizophyllum commune. Current Microbiology 8, 67-71.

VOGEL, H. J. (1956). A convenient growth medium for Neurospora (Medium N). Microbial Genetics Bulletin $13,42-44$

WAKAE, O. \& MATSUURA, K. (1975). Characteristics of validamycin as a fungicide for Rhizoctonia disease control. Review of Plant Protection Research 8, 81-92.

Wessels, J. G. H., Sietsma, J. H. \& SonNenberg, A. S. M. (1983). Wall biosynthesis and assembly during hyphal morphogenesis in Schizophyllum commune. Journal of General Microbiology 129, 1607-1616.

Yoshida, M., Moriya, S. \& VesUGI, Y. (1984). Observations of transmethylation from methionine into choline in the intact mycelia of Pyricularia oryzae by ${ }^{13} \mathrm{C}$ NMR under the influence of fungicides. Journal of Pesticide Science 9, 703-708.

Zsindeley, A., Kiss, A., Schablik, M., SZabolcs, M. \& SzABO, G. (1983). Possible role of a regulatory gene product upon the myo-inositol-1-phosphate synthase production in Neurospora crassa. Biochimica et biophysica acta 741, 273-278. 